\title{
Retraction Note to: A Resource Allocation Algorithm Based on Game Theory in UDN
}

Changjun Chen, Jianxin Dai, Chonghu Cheng, and Zhiliang Huang

\author{
Retraction Note to: \\ Chapter "A Resource Allocation Algorithm Based on Game \\ Theory in UDN" in: X. Gu et al. (Eds.): Machine Learning \\ and Intelligent Communications, LNICST 226, \\ https://doi.org/10.1007/978-3-319-73564-1_45
}

The authors have retracted this conference chapter [1] because it shows significant overlap with a previously published chapter [2]. All authors agree to the retraction.

[1] Chen C., Dai J., Cheng C., Huang Z. (2018) A Resource Allocation Algorithm Based on Game Theory in UDN. In: Gu X., Liu G., Li B. (eds) Machine Learning and Intelligent Communications. MLICOM 2017. Lecture Notes of the Institute for Computer Sciences, Social Informatics and Telecommunications Engineering, vol 226. Springer, Cham

[2] Y. Liu, Y. Wang, Y. Zhang, R. Sun and L. Jiang, "Game-theoretic hierarchical resource allocation in ultra-dense networks," 2016 IEEE 27th Annual International Symposium on Personal, Indoor, and Mobile Radio Communications (PIMRC), Valencia, 2016, pp. 1-6. https://doi.org/10.1109/PIMRC.2016.7794819

The retracted version of this chapter can be found at https://doi.org/10.1007/978-3-319-73564-1_45

(C) ICST Institute for Computer Sciences, Social Informatics and Telecommunications Engineering 2019 